Saudi Journal of Medicine

Abbreviated Key Title: Saudi J Med ISSN 2518-3389 (Print) | ISSN 2518-3397 (Online) Scholars Middle East Publishers, Dubai, United Arab Emirates Journal homepage: https://saudijournals.com

\title{
A Confusing Presentation of Acute Chest Pain: Case Report
}

\author{
Salhah Saleh Alsulami ${ }^{*}$, Sara Hani Alhajri ${ }^{2}$, Shahad Musleh Alyaslami ${ }^{2}$, Mohmad Magdi Atta \\ ${ }^{1}$ Assistant Professor, Rabigh College of Medicine, King Abdulaziz University, Jeddah, Saudi Arabia \\ ${ }^{2} 4$ th Year Medical Student at Rabigh College of Medicine, King Abdulaziz University, Jeddah Saudi, Arabia \\ ${ }^{3}$ Professor at Rabigh College of Medicine, King Abdulaziz University, Jeddah, Saudi Arabia
}

DOI: $10.36348 /$ sjm.2021.v06i02.001

| Received: 09.01.2021 | Accepted: 20.01.2021 | Published: 03.02.2021

*Corresponding Author: Salhah Saleh Alsulami

\section{Abstract}

Introduction and Research Problem: The resemblance between the presentations of acute myocarditis and myocardial infarction poses a great challenge regarding diagnosis and management. Materials and Methods: This study describes the case of a young man aged 25 who had acute retrosternal pain, elevated cardiac markers and electrocardiographic ST-T changes. The patient is 15 pack-year smoker and has positive history of coronary artery disease of his father and his eldest brother. The patient underwent coronary angiography, which revealed normal coronary arteries. Summary of Results: The diagnosis of myopericarditis was made on consideration of his fever, sore throat and body ache for 5 days prior to chest pain, elevated cardiac markers, electrocardiographic ST-T changes but only after the exclusion of acute coronary syndrome by a normal coronary angiogram. Conclusion and Recommendations: Myopericarditis can present with a wide variety of findings. Some of those findings are also characteristic of acute coronary syndrome. This might make the diagnosis confusing. However, even if the patient is young and the presentation is consistent with myopericarditis, still it is important to exclude the diagnosis of acute coronary syndrome by performing a coronary angiogram before settling on the diagnosis of myopericarditis.

Keywords: Myocarditis, myopericariditis, acute myocardial infarction, atypical chest pain, typical chest pain, chest pain in young man, acute viral pericarditis.

Copyright (C) 2021 The Author(s): This is an open-access article distributed under the terms of the Creative Commons Attribution 4.0 International License (CC BY-NC 4.0) which permits unrestricted use, distribution, and reproduction in any medium for non-commercial use provided the original author and source are credited.

\section{INTRODUCTION}

Acute myocarditis can present with a wide variety of features and some of which are found also in acute coronary syndrome. Therefore the differential diagnosis can be very challenging. Usually a detailed history, physical examination and ECG findings are sufficient for diagnosis [1].

To make the differentiation even more challenging is the fact that serum troponin which is a marker of cardiac myocyte damage and is typically used to assess for myocardial infarction in patients presenting with acute coronary syndrome is also found elevated in many conditions with myocardial damage other than myocardial infarction eg. cardiomyopathy, myocarditis, or pulmonary embolism [2].

Acute myocarditis is an acute inflammation of the myocardium. In acute pericarditis there is often associated myocarditis to some extent, although not always. But in clinical practice, usually pericarditis and myocarditis coexist due to the common etiologic factors they share [3]. In a study that followed up myocarditis and pericarditis cases for three years, there was no difference in terms of global complications but mortalities were higher in cases with myopericarditis [4]. The etiologies may be idiopathic, infectious or noninfectious. Most of the infectious causes are viral eg coxsackie virus. But infectious causes can be bacterial eg. Mycobacterium tuberculosis, fungal eg. Histoplasma and parasitic eg. Toxoplasma. Noninfectious cases include drugs like procainamide and isoniazid, systemic diseases (SLE, uremia) [5].

Pericarditis can be diagnosed when the patient presents with the characteristic chest pain that is typically retrosternal, pleuritic like, which worsens on lying flat, and is relieved by assuming the leaning forward sitting position. This pain is accompanied with tachycardia, dyspnea, fever, elevated CRP, frictional rub, pericardial effusion by echocardiography or CT and when cardiac biomarkers are positive [6].

\section{CASE REPORT}

A 25-year-old man presented to ER in King Abdulaziz University Hospital (KAUH) with 2 days history of retrosternal chest pain radiated to left arm. The pain started suddenly at rest; it is sharp, severe enough to interfere with sleep. It is worsening with 
cough, breathing and relieved by leaning forward. It is associated with palpitation, fever and shortness of breath. He has fever, sore throat and body ache for 5 days prior to chest pain. The patient is 15 packs-year smoker. He has positive history of coronary artery disease on his father and his eldest brother.

On examination: HR 107bpm, BP:120/63, Oxygen saturation $100 \%$. Cardiovascular: normal $1^{\text {st }}$ and $2^{\text {nd }}$ heart sound and no added sounds. Chest was equal air entry. There is no lower limb edema.

\section{Investigations}

WBC: 12.1 (normal $4.5-11.5 \mathrm{k} / \mathrm{ul}$ ),

Lymphocyte: 22.2(normal 20-45\%),

Neutrophil: 86.7 (normal50-70\%)

Troponin I: 17 (normal 0.02-0.04 ug/ml)

Creatine kinase: 1701(normal 26-308 IU/L)

Lactate dehydrogenase: 408(normal100- $240 \mathrm{U} / \mathrm{L}$ )

Aspartate aminotransferase: 121(normal 15-37U/L)

LDL: 2.1(normal 0-3.5 nmol/L)
HDL: 0.67 (normal 0.9-1.5nmol/L)

Total cholesterol: 3.2(normal 0-5.2 nmol/L)

TG: 1.04 (normal0.3- $2.3 \mathrm{nmol} / \mathrm{L}$ )

HIV 1,2: -ve

Hepatitis Bs Ag: -ve

Hepatitis C antibodies: -ve

\section{Management}

The patient was admitted to coronary care unit as a case of myopericarditis. The patient stayed for 3 days. He was treated with ibuprofen, bisoprolol and paracetamol and colchicine.

\section{Follow Up/Outcome}

Patient was seen in the cardiology clinic 3 weeks after discharge. All his symptoms had completely resolved.

\section{RESULTS}

Results are shown in Figures 1, 2 and 3.

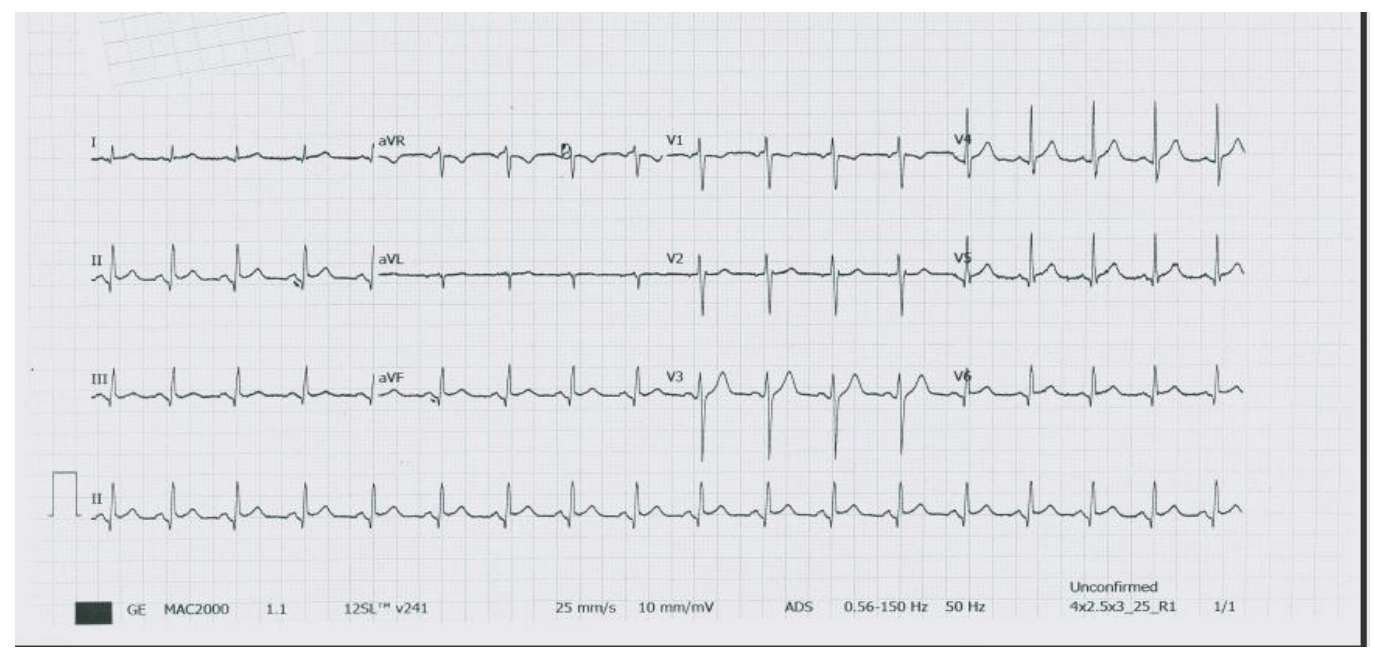

Fig-1: ECG: shows concave ST elevation in leads II, III, aVF, V5 and V6. PR depression on the same leads and sinus tachycardia

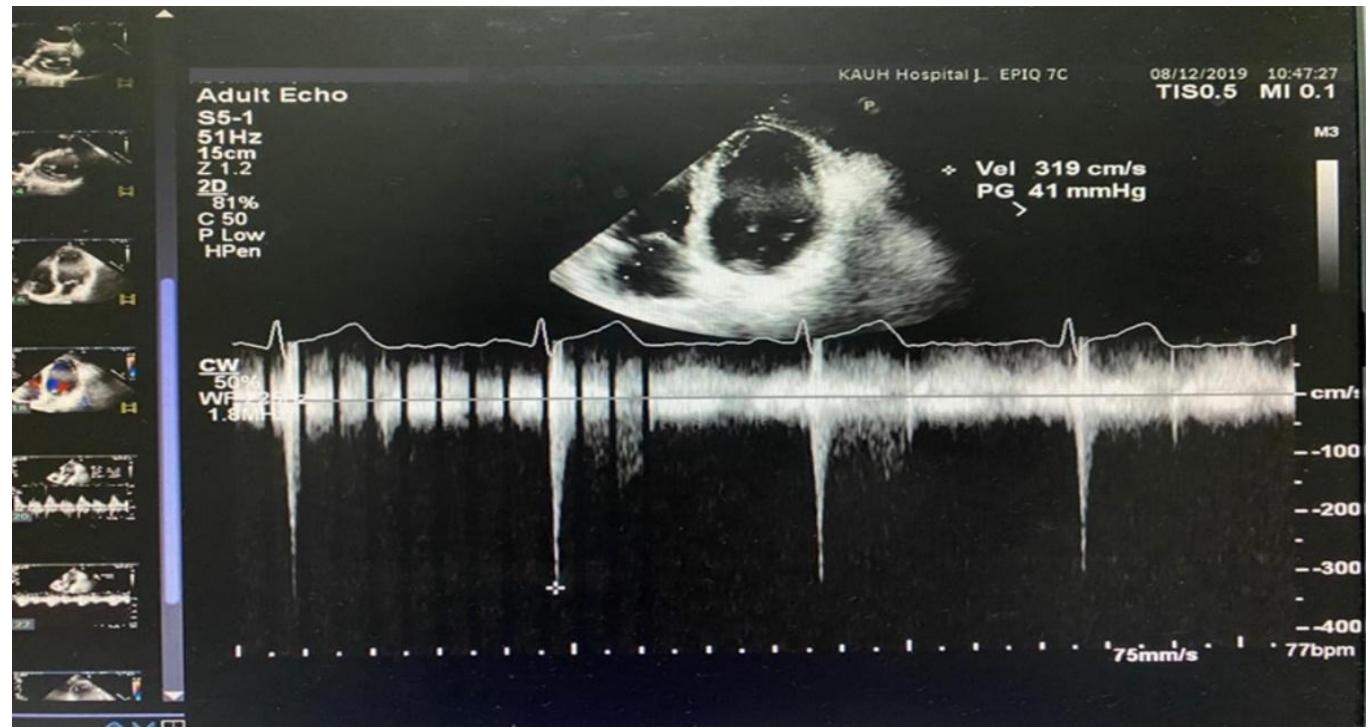

Fig-2: Echocardiography: done during admission which shows normal left and right ventricles size and function and estimated EF60-65\%. There was no pericardial effusion 

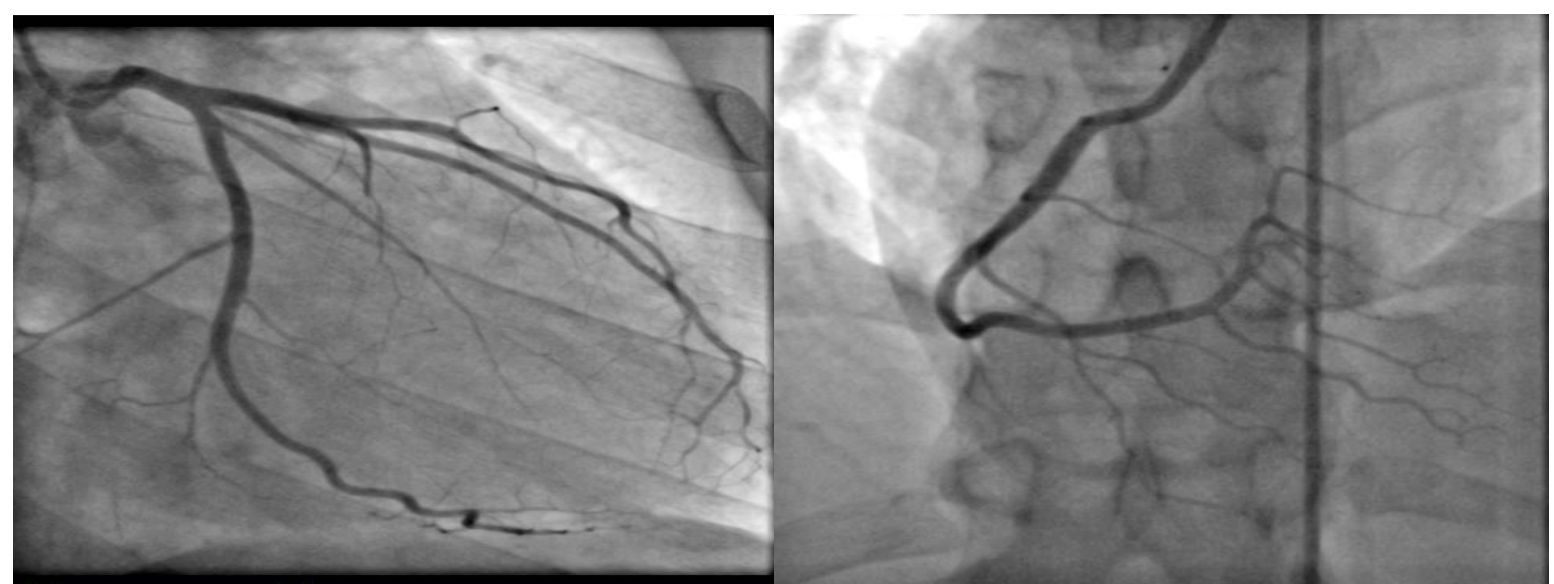

Fig-3: Diagnostic coronary angiography: normal coronary arteries, no significant disease

\section{DISCUSSION}

The presentation of chest pain together with ECG changes and raised markers of myocardial damage are characteristic of pericarditis and myocarditis respectively. Such findings can often lead to diagnostic difficulties as they are nonspecific. Myocardial infarction for example commonly presents in a similar fashion with ST-elevation. It is particularly critical to differentiate between myopericarditis and myocardial as adverse side effects can occur, on one hand, if reperfusion therapy for myocardial infarction is administered for a patient with acute pericarditis, or on the other hand if the diagnosis of acute myocardial infarction is missed and reperfusion therapy is not instituted [7].

Viral myocarditis can present with a wide variety of features some of which may be confused with those of acute coronary syndrome leading to difficulty in the diagnosis and management lines. Moreover the natural course of acute myopericarditis is markedly heterogeneous, ranging from self-limiting disease to cardiopulmonary collapse or even death. Therefore it is necessary in an acute setting that coronary angiography be performed to exclude acute coronary syndrome [8].

This is still true even if the patient is young and presenting with a picture of myocarditis due to the high risks of morbidity and mortality if providing systemic anticoagulation and revascularization was delayed and the case turned out to be Coronary Artery Disease [2].

Very recently, in 2020, viral myocarditis presented within the context of COVID- 19 infection with chest pain, dyspnea, hypotension. Chest $\mathrm{x}$ ray showed marked cardiomegaly, pneumonia and pleural effusion. There was ST-Segment elevation and markedly elevated markers of myocardial injury. Consequently an emergency CT coronary angiography was done and revealed no coronary affection. The case was saved by the use of glucocorticoid as an antiinflammatory with human immunoglobulin therapy [9].
In our patient additional diagnostic challenge was met as he is smoker and has a strong family history of coronary artery disease. That is why coronary angiography was essential to rule out acute coronary artery disease and when it turned out to be normal the decision to manage as myopericarditis was settled. His cardiac condition started after flu like symptoms and it was self-limited.

\section{CONCLUSION}

The great similarity between myocarditis and acute coronary syndrome regarding the clinical presentation, ECG finding and rise of troponin poses a diagnostic challenge. The different management lines and the risk of serious side effects expected if one therapeutic modality was mistakenly instituted instead of the other complicates this difficulty. Therefore it is of extreme importance that clinicians rule out coronary artery disease by angiography before deciding on a treatment modality.

\section{REFERENCES}

1. Yildirim E, Bugan B, Koklu M, Gormel S, Celik M. Acute myocarditis mimicking myocardial infarction can misdirect the diagnostic approach. International Journal of the Cardiovascular Academy. 2016 Mar 1;2(1):12-5.

2. Tandon V, Kumar M, Mosebach CM, Tandon AA. Severe Coronary Artery Disease Disguised as Myocarditis. Cureus. 2019 Feb;11(2):e4159.

3. Imazio M, Trinchero R. Myopericarditis: etiology, management, and prognosis. International journal of cardiology. 2008 Jun 23;127(1):17-26.

4. Machado S, Roubille F, Gahide G, VernhetKovacsik H, Cornillet L, Cung TT, SportouchDukhan C, Raczka F, Pasquié JL, Gervasoni R, Macia JC. Can troponin elevation predict worse prognosis in patients with acute pericarditis?. InAnnales de cardiologie et d'angeiologie 2010 Feb 1 (Vol. 59, No. 1, pp. 1-7). Elsevier Masson.

5. Yugandhar RM, Krishna MB. Myopericarditis. Last Update: December 16, 2019. StatPearls https://www.ncbi.nlm.nih.gov/books/NBK534776/ 
6. Wang K, Asinger RW, Marriott HJ. ST-segment elevation in conditions other than acute myocardial infarction. New England Journal of Medicine. 2003 Nov 27;349(22):2128-35.

7. Lee PT, See CK, Chiam PT, Lim ST. Electrocardiographic changes in acute perimyocarditis. Singapore medical journal. 2015 Jan;56(1):e1-3.

8. Lee WS, Lee KJ, Kwon JE, Oh MS, Kim JE, Cho EJ, Kim CJ. Acute viral myopericarditis presenting as a transient effusive-constrictive pericarditis caused by coinfection with coxsackieviruses A4 and B3. The Korean journal of internal medicine. 2012 Jun;27(2):216.

9. Hongde H, Fenglian M, Xin W, Yuan F. European Heart Journal, ehaa190 https://doi.org/10.1093/eurheartj/ehaa190 Published: 16 March 2020. 